\title{
Required amounts of medium and fertilizer for potted culture of zucchini
}

\author{
Hiromi Ikeura*, Takahiko Tokuda, Yasuyoshi Hayata \\ School of Agriculture, Meiji University, Kanagawa, Japan; ${ }^{*}$ Corresponding Author: hikeura@meiji.ac.jp
}

Received 23 July 2012; revised 29 August 2012; accepted 13 September 2012

\begin{abstract}
In Japan, zucchini culture has yet to get underway, and the current costs of zucchini can be attributed to damage from soil-borne disease and the unstable yields due to seasonal change of female flowers. Eradication of these problems will lead to stable supply and a consequent price reduction of zucchini fruits. We previously clarified the efficacy of potted culture as a new culture method for zucchini, but potted culture can be burdensome as the weak water and nutrient retention capacity of the medium warrants its regular replacement. To solve this problem, in this study, we investigated the blend ratio for mixing rice husk charcoal with peat and the amount of fertilizer required for potted culture of zucchini. Results revealed no significant differences in the length of the largest leaf, total number of flowers, number of female flowers, and the ratio of female flowers to total flowers with different blend ratios of rice husk charcoal to peat. However, the number of harvested fruits increased with higher ratios of rice husk charcoal to peat and was highest at 80:20. The length of the largest leaf increased with increased amounts of fertilizer, with the best response was at $200 \mathrm{~g}$. No significant differences were noted between the ratio of female flowers to total flowers in any treatments. In addition, the number of harvested fruits was highest with $160 \mathrm{~g}$ and $200 \mathrm{~g}$ of fertilizer. Taken together, the optimal blend ratio of rice husk charcoal to peat is $80: 20$, and the optimal amount of fertilizer with this ratio is $160 \mathrm{~g}$.
\end{abstract}

Keywords: Zucchini; Rice Husk Charcoal; Peat; Potted Culture; Fertilizer

\section{INTRODUCTION}

In Japan, large-scale zucchini culture has yet to get underway, and the current costs of zucchini makes wide- spread distribution prohibitive. The high cost of zucchini can be attributed to damage from soil-borne disease and the unstable yields due to seasonal change of female flowers. Eradication of these problems will lead to stable supply and a consequent price reduction of zucchini fruits, which will open the door for zucchini business prospects.

Generally, zucchini is produced open culture, but the culture methods and cultivars are not established due to difference environmental condition in some areas. Especially, planting density in zucchini culture is important to prevent soil-borne disease. In fact, interstrain used in zucchini culture was from $50 \mathrm{~cm}$ to $100 \mathrm{~cm}$, and different by producers or areas. There are many reports about study to demonstrate optimum interstrain and cultivar in zucchini culture [1].

We previously clarified the efficacy of potted culture as a new culture method for zucchini [2]. However, potted culture can be burdensome as the weak water and nutrient retention capacity of the medium warrants its regular replacement. To solve this problem, we considered using rice husk charcoal and peat moss as a medium as both are organic materials with a high cation exchange capacity and are often used as a medium in soilless culture [3-9]. Rice husk charcoal and peat moss have been studied for recycling and the subsequent reclamation of solid and organic residues produced in agriculture, faming, forestry, and industry, and they are also being successfully used as container media for ornamental plant production $[9,10]$. In addition, these organic materials are available for compost after use and are easily applied in the field $[10,11]$. In particular, rice husk charcoal is made from burning rice husks, which are generated from harvested rice, the principal food in Japan and Thailand [12]. In addition, rice husks are typically utilized as a medium for ornamental crops (e.g., Chrysanthemum morifolium) and as soil cover for protecting rice in nurseries by covering with oil paper. Such uses are common because rice husk charcoal is affordable, has high cation exchange and aeration properties, has good moisture retentiveness due to its porosity, and mixes well with other substances $[4,13,14]$. Moreover, Inden and Torres (2004) [14] re- 
ported an increase in yield and total soluble solid content in tomato when using coconut coir or perlite plus carbonized rice hulls as a growth substrate. Islam (2008) [15] described focusing on good aeration and lightweight properties and thereby found rise husk charcoal to be a suitable medium for soilless culture. Peat moss, which has high moisture and nutrient retentiveness as well as a high buffering ability, is particularly utilized as a growth medium for strawberry culture $[5,16]$.

In this study, we investigated the effects of different ratios of rice husk charcoal to peat moss as a medium as well as the optimal amount of fertilizer required for zucchini potted culture.

\section{MATERIALS AND METHODS}

\subsection{Culture Conditions}

Zucchini seeds "Diner" (Takii Co. Ltd., Kyoto, Japan) were planted in a plastic tray containing propagation medium (Coop Chemical Co. Ltd., Tokyo, Japan) and vermiculite (Showa vermiculite Co. Ltd., Kanagawa, Japan) (1:1). A hydroponic fertilizer (Otsuka house No. 1 and No. 2) diluted 2-fold in tap water (as a standard concentration) was added for propagation. Interstrain and interrow spaces after settled planting were $60 \mathrm{~cm}$ and 80 $\mathrm{cm}$, respectively. Agrisheet was laid out in a greenhouse as the pots should not have contact with regular soil. Rice husk charcoal and peat were purchased from Berry's life Co. (Kagawa, Japan) and Nichias Co. (Tokyo, Japan). Irrigation after settled planting was conducted at $3 \mathrm{~L} \cdot \operatorname{pot}^{-1}$ at 7 am daily.

\subsection{Blend Ratio of Rice Husk Charcoal to Peat}

Culture was conducted in a greenhouse at Meiji University from July 6, 2008 to October 28, 2008. Zucchini seeds were planted in $12-\mathrm{cm}$ plastic pots (height $10 \mathrm{~cm}$ ) containing propagation medium, and seedlings were cultured until developing 7 leaves. The plants were then settled in $30.5-\mathrm{cm}$ plastic pots (height $30.5 \mathrm{~cm}$; medium volume, 15 L; Kaneko Seed Co. Ltd., Gunma, Japan) on July 28, 2008.

The following four blend ratios of rice husk charcoal to peat were set: 80:20,60:40, 40:60, and 20:80. Eighty grams of delayed release fertilizer, Ecolong 424 (N:P:K = 14:12:13, JCAM AGRI. Co., Ltd., Tokyo, Japan), and $210 \mathrm{~g}$ of compost (Fujimi group, Sizuoka, Japan) were added, and the $\mathrm{pH}$ of each medium was adjusted to the same level ( $\mathrm{pH}$ 6.5) by adding $7 \mathrm{~g}$ of magnesium lime (Aiko Sekkai Co., Tochigi, Japan) for 80:20 and 60:40 and $120 \mathrm{~g}$ of magnesium lime for 40:60 and 20:80. After adding each medium, fertilizer, compost, and water were mixed in a soil mixer and drained. All experiments were replicated 3 times, with 10 plants per replicate.

\subsection{Amount of Fertilizer}

Culture was conducted from August 11, 2009 to December 15, 2009 under the same conditions with different blend ratios of rice husk charcoal to peat. Plants were settled in 30.5-cm plastic pots on August 29, 2009. The following four amounts of fertilizer were set: $80 \mathrm{~g}, 120 \mathrm{~g}$, $160 \mathrm{~g}$, and $200 \mathrm{~g}$. Based in the results of the above experiments, the most appropriate ratio of medium was mixed to a volume of $15 \mathrm{~L}$. Other applied fertilizers were $210 \mathrm{~g}$ of compost and $7 \mathrm{~g}$ of magnesium lime. All experiments were replicated 3 times, with 10 plants per replicate.

\section{Growth, Pollination, and Harvest}

The length of the largest leaf, the total number of flowers, and the number of female flowers were measured once every 7 days to establish the female flower to total flower ratio. Pollination was conducted at $8-9$ am, and fruits were harvested at a size with a market value $(16-19 \mathrm{~cm})$.

\subsection{Statistical Analysis}

Data are presented as mean and standard error values, and statistical differences between treatments were tested using the Tukey-Kramer test at $\mathrm{P}<0.05$.

\section{RESULTS AND DISCUSSION}

The effect of different ratios of rice husk charcoal to peat on zucchini growth is shown in Table 1. There was no significant difference in the length of the largest leaf, total number of flowers, number of female flowers, or the ratio of female flowers to the total number of flowers. The number of harvested fruits per pot was 2.8 with $20: 80,2.7$ with $40: 60,4.2$ with $60: 40$, and 4.8 with $80: 20$, respectively; an obvious increasing trend with increasing rice husk charcoal (Figure 1). Endo et al. (2006) [16] demonstrated the effects of root zone substrates consisting of coir and peat mixture on the growth and yield of strawberry and indicated that the marketable yield of strawberries increased with decreasing peat ratios. Islam (2008) [15] described that rice husk charcoal gave similar and/or better crop performance and yield of tomatoes than rock wool under high-temperature stress conditions $\left(30^{\circ} \mathrm{C}\right.$ and $35^{\circ} \mathrm{C}$ versus $\left.25^{\circ} \mathrm{C}\right)$, which showed that rice husk charcoal can be used successfully as a growing medium amendment for producing greenhouse tomato as well as other nursery crops. Promchot and Boonprakob (2007) [12] reported that rice husk charcoal was better than agar for embryo culture of nectarines and that it may be a substitute for vermiculite. Thus, rice husk 
Table 1. Effect of different blend ratios of rice husk charcoal to peat on the growth of zucchini.

\begin{tabular}{|c|c|c|c|c|c|c|c|}
\hline \multirow{2}{*}{ Treatment } & & \multicolumn{6}{|c|}{ Days after planting (mean \pm S.E.) } \\
\hline & & 15 days & 22 days & 29 days & 36 days & 43 days & 50 days \\
\hline \multirow{4}{*}{$\begin{array}{l}\text { The length of } \\
\text { largest leaf }(\mathrm{cm})\end{array}$} & $20: 80$ & $71.20 \pm 2.86 \mathrm{a}^{\mathrm{y}}$ & $84.20 \pm 4.29 a$ & $85.20 \pm 1.87 \mathrm{a}$ & $86.70 \pm 2.26 \mathrm{a}$ & $88.80 \pm 4.87 \mathrm{a}$ & $89.44 \pm 5.03 a$ \\
\hline & $40: 60$ & $70.40 \pm 4.38 \mathrm{a}$ & $85.60 \pm 3.50 \mathrm{a}$ & $87.30 \pm 2.91 \mathrm{a}$ & $88.70 \pm 2.79 a$ & $89.70 \pm 2.41 \mathrm{a}$ & $91.10 \pm 3.14 \mathrm{a}$ \\
\hline & $60: 40$ & $70.80 \pm 2.66 \mathrm{a}$ & $84.10 \pm 4.56 \mathrm{a}$ & $86.60 \pm 5.06 \mathrm{a}$ & $88.33 \pm 4.80 \mathrm{a}$ & $90.56 \pm 3.21 \mathrm{a}$ & $91.56 \pm 3.00 \mathrm{a}$ \\
\hline & $80: 20$ & $68.20 \pm 4.76 a$ & $80.40 \pm 5.62 \mathrm{a}$ & $84.80 \pm 5.55 a$ & $87.30 \pm 4.81 \mathrm{a}$ & $88.30 \pm 4.90 \mathrm{a}$ & $89.10 \pm 5.03 a$ \\
\hline \multirow{4}{*}{$\begin{array}{l}\text { A number of female } \\
\text { flowers (flower) }\end{array}$} & $20: 80$ & $3.00 \pm 0.94 \mathrm{a}$ & $5.80 \pm 0.79 \mathrm{a}$ & $8.50 \pm 0.71 \mathrm{a}$ & $12.20 \pm 1.75 \mathrm{a}$ & $15.80 \pm 1.87 \mathrm{a}$ & $20.33 \pm 1.87 \mathrm{a}$ \\
\hline & $40: 60$ & $2.90 \pm 0.57 \mathrm{a}$ & $5.60 \pm 0.70 \mathrm{a}$ & $8.20 \pm 0.63 \mathrm{a}$ & $12.10 \pm 1.20 \mathrm{a}$ & $15.50 \pm 1.18 \mathrm{a}$ & $18.70 \pm 1.19 \mathrm{a}$ \\
\hline & $60: 40$ & $3.00 \pm 1.05 \mathrm{a}$ & $5.40 \pm 1.11 \mathrm{a}$ & $7.80 \pm 2.10 \mathrm{a}$ & $11.78 \pm 1.72 \mathrm{a}$ & $15.11 \pm 2.37 \mathrm{a}$ & $18.56 \pm 2.35 \mathrm{a}$ \\
\hline & $80: 20$ & $3.60 \pm 1.07 \mathrm{a}$ & $6.20 \pm 0.92 \mathrm{a}$ & $8.90 \pm 1.66 \mathrm{a}$ & $13.00 \pm 2.11 \mathrm{a}$ & $16.50 \pm 2.55 \mathrm{a}$ & $19.90 \pm 3.05 \mathrm{a}$ \\
\hline \multirow{4}{*}{$\begin{array}{l}\text { Total number of } \\
\text { flowers (flower) }\end{array}$} & $20: 80$ & $20.90 \pm 0.88 \mathrm{a}$ & $28.40 \pm 0.97 \mathrm{a}$ & $34.00 \pm 1.25 \mathrm{a}$ & $42.70 \pm 1.42 \mathrm{a}$ & $50.60 \pm 1.78 \mathrm{a}$ & $57.89 \pm 1.96 a$ \\
\hline & $40: 60$ & $20.10 \pm 0.57 \mathrm{a}$ & $27.30 \pm 0.67 \mathrm{a}$ & $33.80 \pm 1.03 \mathrm{a}$ & $42.30 \pm 0.82 \mathrm{a}$ & $50.10 \pm 0.88 \mathrm{a}$ & $57.40 \pm 1.43 \mathrm{a}$ \\
\hline & $60: 40$ & $21.00 \pm 1.25 \mathrm{a}$ & $29.00 \pm 1.15 \mathrm{a}$ & $35.50 \pm 1.58 \mathrm{a}$ & $43.00 \pm 1.73 a$ & $51.44 \pm 1.88 \mathrm{a}$ & $57.22 \pm 1.79 a$ \\
\hline & $80: 20$ & $21.20 \pm 1.03 \mathrm{a}$ & $28.50 \pm 5.62 \mathrm{a}$ & $35.30 \pm 3.09 \mathrm{a}$ & $43.00 \pm 3.02 \mathrm{a}$ & $51.40 \pm 2.95 \mathrm{a}$ & $58.00 \pm 3.97 \mathrm{a}$ \\
\hline \multirow{4}{*}{$\begin{array}{l}\text { The ratio of female } \\
\text { flowers to the total } \\
\text { number of flowers } \\
(\%)\end{array}$} & $20: 80$ & $14.35 \pm 1.08 \mathrm{a}$ & $20.42 \pm 0.82 \mathrm{a}$ & $25.00 \pm 0.57 \mathrm{a}$ & $28.57 \pm 1.23 \mathrm{a}$ & $31.23 \pm 1.05 \mathrm{a}$ & $35.12 \pm 0.95 \mathrm{a}$ \\
\hline & $40: 60$ & $14.43 \pm 1.00 \mathrm{a}$ & $20.51 \pm 1.04 \mathrm{a}$ & $24.26 \pm 0.61 \mathrm{a}$ & $28.61 \pm 1.45 \mathrm{a}$ & $30.94 \pm 1.35 \mathrm{a}$ & $32.58 \pm 0.83 a$ \\
\hline & $60: 40$ & $14.29 \pm 0.85 \mathrm{a}$ & $18.62 \pm 0.96 \mathrm{a}$ & $21.97 \pm 1.33 \mathrm{a}$ & $27.39 \pm 0.99 \mathrm{a}$ & $29.37 \pm 1.26 \mathrm{a}$ & $32.43 \pm 1.32 \mathrm{a}$ \\
\hline & $80: 20$ & $16.98 \pm 1.04 \mathrm{a}$ & $21.75 \pm 0.16 \mathrm{a}$ & $25.21 \pm 0.54 \mathrm{a}$ & $30.23 \pm 0.70 \mathrm{a}$ & $32.10 \pm 0.86 \mathrm{a}$ & $34.31 \pm 0.77 \mathrm{a}$ \\
\hline \multirow{2}{*}{\multicolumn{2}{|c|}{ Treatment }} & \multicolumn{6}{|c|}{ Days after planting (mean \pm S.E.) } \\
\hline & & 57 day & 64 day & 71 day & 78 day & 85 day & 92 day \\
\hline \multirow{4}{*}{$\begin{array}{l}\text { The length of largest } \\
\text { leaf }(\mathrm{cm})\end{array}$} & $20: 80$ & $89.44 \pm 5.03 a$ & $89.75 \pm 5.26 a$ & $89.75 \pm 5.26 a$ & $89.75 \pm 5.26 \mathrm{a}$ & $89.75 \pm 5.26 a$ & $89.75 \pm 5.26 a$ \\
\hline & $40: 60$ & $91.60 \pm 3.50 \mathrm{a}$ & $91.60 \pm 3.50 \mathrm{a}$ & $91.60 \pm 3.50 \mathrm{a}$ & $91.60 \pm 3.50 \mathrm{a}$ & $91.22 \pm 3.49 \mathrm{a}$ & $91.22 \pm 3.49 \mathrm{a}$ \\
\hline & $60: 40$ & $91.50 \pm 2.78 \mathrm{a}$ & $91.50 \pm 2.78 \mathrm{a}$ & $91.43 \pm 2.99 \mathrm{a}$ & $91.43 \pm 2.99 \mathrm{a}$ & $91.43 \pm 2.99 \mathrm{a}$ & $91.43 \pm 2.99 \mathrm{a}$ \\
\hline & $80: 20$ & $89.10 \pm 5.30 \mathrm{a}$ & $89.67 \pm 5.29 \mathrm{a}$ & $89.67 \pm 5.29 a$ & $89.67 \pm 5.29 \mathrm{a}$ & $89.67 \pm 5.29 \mathrm{a}$ & $89.67 \pm 5.29 a$ \\
\hline \multirow{4}{*}{$\begin{array}{l}\text { A number of female } \\
\text { flowers (flower) }\end{array}$} & $20: 80$ & $22.89 \pm 2.52 \mathrm{a}$ & $25.50 \pm 2.73 \mathrm{a}$ & $27.00 \pm 3.38 \mathrm{a}$ & $28.88 \pm 4.22 \mathrm{a}$ & $30.50 \pm 4.78 \mathrm{a}$ & $31.38 \pm 4.78 \mathrm{a}$ \\
\hline & 40:60 & $20.70 \pm 2.21 \mathrm{a}$ & $22.70 \pm 1.64 a$ & $24.10 \pm 1.79 a$ & $26.10 \pm 2.13 \mathrm{a}$ & $28.78 \pm 2.64 a$ & $29.00 \pm 2.40 \mathrm{a}$ \\
\hline & $60: 40$ & $20.78 \pm 3.15 \mathrm{a}$ & $22.88 \pm 3.87 \mathrm{a}$ & $24.86 \pm 4.74 a$ & $26.43 \pm 4.72 \mathrm{a}$ & $28.43 \pm 4.93 a$ & $30.29 \pm 4.57 \mathrm{a}$ \\
\hline & $80: 20$ & $21.80 \pm 2.94 \mathrm{a}$ & $24.00 \pm 3.20 \mathrm{a}$ & $25.67 \pm 3.46 \mathrm{a}$ & $27.56 \pm 3.54 \mathrm{a}$ & $29.22 \pm 4.38 \mathrm{a}$ & $29.67 \pm 3.61 \mathrm{a}$ \\
\hline \multirow{4}{*}{$\begin{array}{l}\text { Total number of } \\
\text { flowers (flower) }\end{array}$} & $20: 80$ & $62.89 \pm 2.62 \mathrm{a}$ & $67.13 \pm 2.36 \mathrm{a}$ & $70.75 \pm 2.49 a$ & $74.25 \pm 4.65 \mathrm{a}$ & $77.63 \pm 5.66 \mathrm{a}$ & $80.00 \pm 6.61 \mathrm{a}$ \\
\hline & $40: 60$ & $62.80 \pm 1.55 \mathrm{a}$ & $66.60 \pm 1.65 \mathrm{a}$ & $69.80 \pm 2.20 \mathrm{a}$ & $74.20 \pm 3.33 \mathrm{a}$ & $79.44 \pm 2.96 a$ & $83.22 \pm 2.82 \mathrm{a}$ \\
\hline & $60: 40$ & $63.25 \pm 2.12 \mathrm{a}$ & $67.25 \pm 2.25 \mathrm{a}$ & $71.00 \pm 2.00 \mathrm{a}$ & $74.71 \pm 2.06 a$ & $78.00 \pm 2.52 \mathrm{a}$ & $81.14 \pm 2.54 a$ \\
\hline & $80: 20$ & $61.90 \pm 5.02 \mathrm{a}$ & $66.67 \pm 2.60 \mathrm{a}$ & $69.89 \pm 2.67 \mathrm{a}$ & $73.67 \pm 2.92 \mathrm{a}$ & $77.22 \pm 2.82 \mathrm{a}$ & $80.44 \pm 3.05 \mathrm{a}$ \\
\hline \multirow{4}{*}{$\begin{array}{l}\text { The ratio of female } \\
\text { flowers to the total } \\
\text { number of flowers } \\
(\%)\end{array}$} & $20: 80$ & $36.40 \pm 0.96 \mathrm{a}$ & $37.99 \pm 1.16 \mathrm{a}$ & $38.16 \pm 1.36 \mathrm{a}$ & $38.89 \pm 0.91 \mathrm{a}$ & $39.29 \pm 0.85 \mathrm{a}$ & $39.22 \pm 0.72 a$ \\
\hline & $40: 60$ & $32.96 \pm 1.43 \mathrm{a}$ & $34.08 \pm 0.99 a$ & $34.53 \pm 0.81 \mathrm{a}$ & $35.18 \pm 0.64 \mathrm{a}$ & $36.22 \pm 0.89 a$ & $34.85 \pm 0.85 a$ \\
\hline & $60: 40$ & $32.85 \pm 1.49 a$ & $34.01 \pm 1.72 \mathrm{a}$ & $35.01 \pm 2.37 \mathrm{a}$ & $35.37 \pm 2.29 a$ & $36.45 \pm 1.96 a$ & $37.32 \pm 1.80 \mathrm{a}$ \\
\hline & $80: 20$ & $35.22 \pm 0.58 \mathrm{a}$ & $36.00 \pm 1.23 \mathrm{a}$ & $36.72 \pm 1.30 \mathrm{a}$ & $37.41 \pm 1.21 \mathrm{a}$ & $37.84 \pm 1.55 \mathrm{a}$ & $36.88 \pm 1.18 \mathrm{a}$ \\
\hline
\end{tabular}

The ratio of female flowers to the total number of flowers $=$ a number of female/total number of flowers $\times 100$; ${ }^{\mathrm{y}}$ Different letter indicate statistical differences between treatments were tested using the Turkey-Kramer test at $\mathrm{P}<0.05(\mathrm{n}=10)$. 


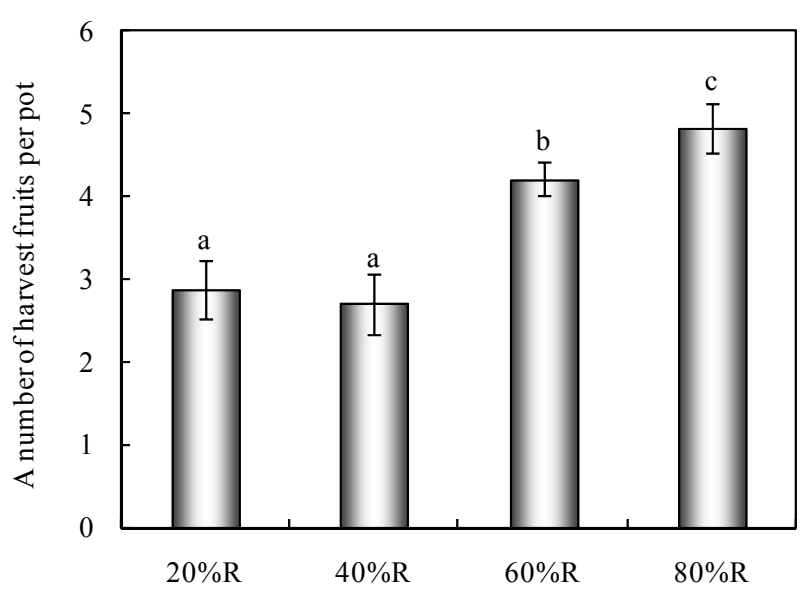

Figure 1. Effect of different blend ratios of rice husk charcoal to peat on the number of harvested zucchini fruits Vertical bars represent one standard deviation of the mean. Different letters indicate significant difference at the 5\% level according to the Tukey-Kramer test between treatments $(n=10)$.

charcoal has various effects on plant variety and growth stages, but its most prominent effect is as a culture medium. In addition, variety-specific responses should be considered when giving recommendations for adding the optimum proportion of organic material amendments to horticultural potting substrate [17].

It is considered that the $80: 20$ medium drained well because the amount of peat (which retains water) was decreased and because zucchini is better suited to welldrained soil. Oshio et al. (1981) [4] discussed that rice husk charcoal has a porous structure which contributes to aeration and water retention, which in turn enhances water and nutrient (especially potassium and phosporous) retention. These factors suggest that the number of harvested fruits increased because sufficient nutrients were available as a result of the enhanced water and nutrient retention in the medium.

Potted culture of zucchini is essential to be cheaper cost of medium due to consisting mostly medium cost. In Japan, rice is the staple crop, and rice husk charcoal is abundant and affordable [14]. Therefore, the cost of rice husk charcoal medium for potted culture will enable feasible cultivation options for zucchini.

The effect of different amounts of applied fertilizer on the growth of zucchini is shown in Table 2. The length of the largest leaf, total number of flowers, and the number of female flowers increased with higher amounts of applied fertilizer and was highest at $200 \mathrm{~g}$. This was thought that a number of nude increased to have great vigor of fruits growth with rise amount of applied fertilizer. There was no significant difference in the ratio of female flowers to the total number of flowers in any treatments. Temperature is an important external environmental factor for female differentiation of Cucurbita- ceae plants, the family to which zucchini belongs [18]. All experiments in the present study were conducted under the same conditions in the same greenhouse to ensure the same external variables. Thus it can be inferred that the ratio of female flowers to the total number of flowers does not fluctuate.

The effect of different amounts of applied fertilizer on the number of harvested zucchini fruits is shown in Figure 2. The number of harvested fruits per pot was 4.6 with $80 \mathrm{~g}, 6.1$ with $120 \mathrm{~g}, 8.7$ with $160 \mathrm{~g}$, and 8.9 with $200 \mathrm{~g}$, and increased with increasing amounts of fertilizer and was highest with $200 \mathrm{~g}$. There was no significant difference in the number of harvested fruits and the growth of zucchini with $160 \mathrm{~g}$ and $200 \mathrm{~g}$. It was assumed that the nutrient uptake ability of the roots was limited because the roots were restricted by the pot. For effective tomato culture, Zuraiqi and Battilhi (1992) [19] recommended four applications of nitrogen fertilizer per season at a rate of $30 \mathrm{~kg} / \mathrm{ha}$ at two-week intervals. In contrast, Diez et al. (1997) [20] and Eghball and Power (1999) [21] obtained no yield differences between crops grown with compost and those grown with chemical fertilizer. Evanylo et al. (2008) [22] reported that various treatments with organic fertilizer and a commercial agricultural fertilizer did not affect pumpkin or bell pepper growth. Reiners and Riggs (1997) [23] demonstrated the effect of nitrogen application and the variety of pumpkin (Cucurbita pepo L.) marketable yield and found that pumpkin yield was unaffected by 67, 112, and 157 $\mathrm{kg} \cdot \mathrm{N} \cdot \mathrm{ha}^{-1}$. On the other hand, Takemyou et al. (2002) [24] reported that the growth of zucchini and harvested fruits increased in line with increased amounts of applied fertilizer and that $80-160 \mathrm{~g}$ medium from a $10 \mathrm{~L}$ volume is suitable for potted culture of zucchini. In this study,

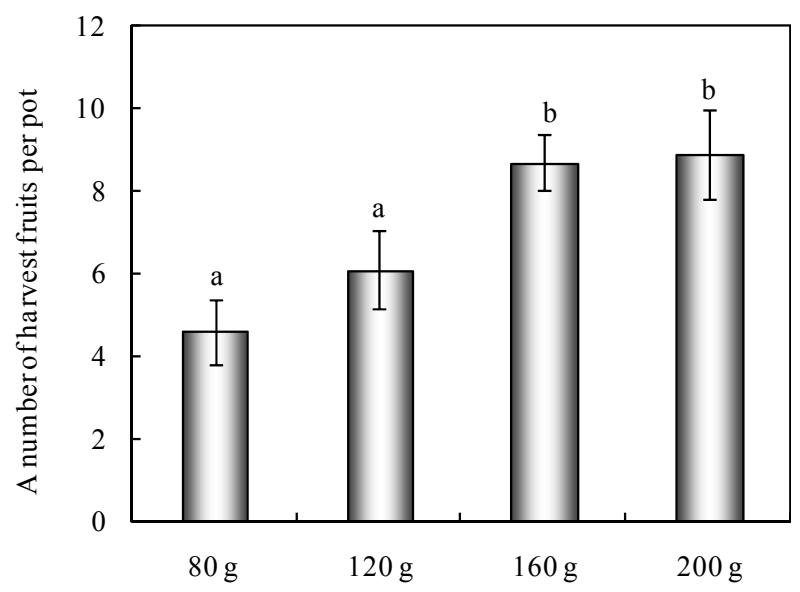

Figure 2. Effect of different amounts of applied fertilizer on the number of harvested zucchini fruits Vertical bars represent one standard deviation of the mean. Different letters indicate significant difference at the $5 \%$ level according to the TukeyKramer test between treatments $(n=10)$. 
Table 2. Effect of different amounts of applied fertilizer on the growth of zucchini.

\begin{tabular}{|c|c|c|c|c|c|c|c|c|c|}
\hline \multirow{2}{*}{\multicolumn{2}{|c|}{ Treatment }} & \multicolumn{8}{|c|}{ Days after planting (mean \pm S.E.) } \\
\hline & & 3 days & 10 days & 17 days & 24 days & 31 days & 38 days & 45 days & 52 days \\
\hline \multirow{4}{*}{$\begin{array}{l}\text { The length of } \\
\text { largest leaf } \\
(\mathrm{cm})\end{array}$} & $80 \mathrm{~g}$ & $28.9 \pm 0.5 \mathrm{a}^{\mathrm{y}}$ & $35.2 \pm 1.1 \mathrm{a}$ & $51.0 \pm 1.5 \mathrm{a}$ & $61.9 \pm 1.9 a$ & $66.3 \pm 2.0 \mathrm{a}$ & $73.0 \pm 2.0 \mathrm{a}$ & $75.8 \pm 2.4 a$ & $78.0 \pm 2.0 \mathrm{a}$ \\
\hline & $120 \mathrm{~g}$ & $30.4 \pm 0.4 \mathrm{a}$ & $34.9 \pm 0.5 \mathrm{a}$ & $49.6 \pm 1.1 \mathrm{a}$ & $62.0 \pm 1.6 \mathrm{ab}$ & $68.7 \pm 1.8 \mathrm{a}$ & $77.6 \pm 2.7 \mathrm{a}$ & $80.7 \pm 2.4 \mathrm{a}$ & $83.1 \pm 2.2 \mathrm{a}$ \\
\hline & $160 \mathrm{~g}$ & $30.1 \pm 0.6 \mathrm{a}$ & $35.2 \pm 0.7 \mathrm{a}$ & $52.2 \pm 1.4 \mathrm{a}$ & $65.5 \pm 1.3 b$ & $75.0 \pm 2.0 \mathrm{a}$ & $81.3 \pm 1.9 \mathrm{ab}$ & $83.4 \pm 1.6 a b$ & $84.6 \pm 1.6 a b$ \\
\hline & $200 \mathrm{~g}$ & $31.3 \pm 1.0 \mathrm{a}$ & $34.7 \pm 0.7 \mathrm{a}$ & $50.0 \pm 1.7 \mathrm{a}$ & $63.6 \pm 3.0 \mathrm{ab}$ & $75.9 \pm 4.0 \mathrm{a}$ & $82.2 \pm 2.8 \mathrm{~b}$ & $84.4 \pm 3.0 \mathrm{~b}$ & $85.3 \pm 3.2 b$ \\
\hline \multirow{4}{*}{$\begin{array}{l}\text { A number of } \\
\text { female flowers } \\
\text { (flower) }\end{array}$} & $80 \mathrm{~g}$ & $6.8 \pm 0.1 \mathrm{a}$ & $11.4 \pm 0.3 \mathrm{a}$ & $17.4 \pm 0.3 \mathrm{a}$ & $22.6 \pm 0.3 \mathrm{a}$ & $30.0 \pm 0.4 a$ & $32.8 \pm 0.4 a$ & $37.1 \pm 0.5 \mathrm{a}$ & $40.9 \pm 0.7 \mathrm{a}$ \\
\hline & $120 \mathrm{~g}$ & $7.0 \pm 0.0 \mathrm{a}$ & $11.5 \pm 0.2 \mathrm{a}$ & $17.4 \pm 0.2 \mathrm{a}$ & $23.0 \pm 0.3 \mathrm{a}$ & $30.2 \pm 0.4 \mathrm{a}$ & $33.9 \pm 0.4 \mathrm{a}$ & $37.8 \pm 0.4 \mathrm{a}$ & $41.0 \pm 0.6 \mathrm{a}$ \\
\hline & $160 \mathrm{~g}$ & $6.9 \pm 0.1 \mathrm{a}$ & $11.7 \pm 0.2 \mathrm{a}$ & $17.5 \pm 0.3 \mathrm{a}$ & $23.4 \pm 0.2 \mathrm{a}$ & $31.0 \pm 0.3 \mathrm{a}$ & $34.6 \pm 0.3 \mathrm{a}$ & $39.3 \pm 0.3 \mathrm{a}$ & $43.0 \pm 0.4 \mathrm{ab}$ \\
\hline & $200 \mathrm{~g}$ & $6.8 \pm 0.1 \mathrm{a}$ & $11.3 \pm 0.2 \mathrm{a}$ & $17.2 \pm 0.2 \mathrm{a}$ & $22.9 \pm 0.5 \mathrm{a}$ & $30.7 \pm 0.6 \mathrm{a}$ & $34.8 \pm 0.6 \mathrm{a}$ & $39.4 \pm 0.7 \mathrm{a}$ & $43.8 \pm 0.7 b$ \\
\hline \multirow{4}{*}{$\begin{array}{l}\text { Total number } \\
\text { of flowers } \\
\text { (flower) }\end{array}$} & $80 \mathrm{~g}$ & $0.0 \pm 0.0 \mathrm{a}$ & $0.1 \pm 0.1 \mathrm{a}$ & $2.2 \pm 0.2 \mathrm{a}$ & $4.9 \pm 0.3 \mathrm{a}$ & $8.0 \pm 0.5 \mathrm{a}$ & $9.2 \pm 0.8 \mathrm{a}$ & $11.3 \pm 0.8 \mathrm{a}$ & $13.0 \pm 1.0 \mathrm{a}$ \\
\hline & $120 \mathrm{~g}$ & $0.0 \pm 0.0 \mathrm{a}$ & $0.6 \pm 0.2 \mathrm{a}$ & $2.1 \pm 0.2 \mathrm{a}$ & $5.1 \pm 0.2 \mathrm{a}$ & $8.1 \pm 0.2 \mathrm{a}$ & $9.7 \pm 0.2 \mathrm{ab}$ & $11.7 \pm 0.4 \mathrm{ab}$ & $13.4 \pm 0.6 \mathrm{ab}$ \\
\hline & $160 \mathrm{~g}$ & $0.0 \pm 0.0 \mathrm{a}$ & $0.3 \pm 0.2 \mathrm{a}$ & $2.4 \pm 0.3 \mathrm{a}$ & $5.4 \pm 0.4 b$ & $8.8 \pm 0.3 \mathrm{a}$ & $10.6 \pm 0.5 b$ & $12.6 \pm 0.6 \mathrm{~b}$ & $14.6 \pm 0.9 b$ \\
\hline & $200 \mathrm{~g}$ & $0.0 \pm 0.0 \mathrm{a}$ & $0.1 \pm 0.1 \mathrm{a}$ & $1.6 \pm 0.2 b$ & $4.6 \pm 0.5 \mathrm{a}$ & $7.9 \pm 0.5 \mathrm{a}$ & $9.4 \pm 0.6 \mathrm{a}$ & $11.7 \pm 0.6 \mathrm{ab}$ & $13.4 \pm 0.9 \mathrm{ab}$ \\
\hline \multirow{4}{*}{$\begin{array}{l}\text { The ratio of } \\
\text { female flowers } \\
\text { to the total } \\
\text { number of } \\
\text { flowers }(\%)\end{array}$} & $80 \mathrm{~g}$ & $0.0 \pm 0.0 \mathrm{a}$ & $1.0 \pm 1.0 \mathrm{a}$ & $12.6 \pm 1.4 \mathrm{a}$ & $21.7 \pm 1.3 \mathrm{a}$ & $26.8 \pm 2.0 \mathrm{a}$ & $27.9 \pm 2.1 \mathrm{a}$ & $30.3 \pm 1.8 \mathrm{a}$ & $31.6 \pm 2.0 \mathrm{a}$ \\
\hline & $120 \mathrm{~g}$ & $0.0 \pm 0.0 \mathrm{a}$ & $5.2 \pm 1.4 \mathrm{a}$ & $12.1 \pm 1.1 \mathrm{a}$ & $22.2 \pm 1.0 \mathrm{a}$ & $26.9 \pm 0.9 \mathrm{a}$ & $28.7 \pm 0.9 \mathrm{a}$ & $31.0 \pm 1.3 \mathrm{a}$ & $32.8 \pm 1.6 \mathrm{a}$ \\
\hline & $160 \mathrm{~g}$ & $0.0 \pm 0.0 \mathrm{a}$ & $2.6 \pm 1.3 \mathrm{a}$ & $13.7 \pm 1.7 \mathrm{a}$ & $23.1 \pm 1.6 \mathrm{a}$ & $28.4 \pm 0.9 \mathrm{a}$ & $30.6 \pm 1.4 \mathrm{a}$ & $32.1 \pm 1.6 \mathrm{a}$ & $33.9 \pm 1.9 \mathrm{a}$ \\
\hline & $200 \mathrm{~g}$ & $0.0 \pm 0.0 \mathrm{a}$ & $0.8 \pm 0.8 \mathrm{a}$ & $9.2 \pm 1.2 \mathrm{a}$ & $19.9 \pm 1.8 \mathrm{a}$ & $25.6 \pm 1.3 \mathrm{a}$ & $26.8 \pm 1.4 \mathrm{a}$ & $29.5 \pm 1.8 \mathrm{a}$ & $30.5 \pm 1.8 \mathrm{a}$ \\
\hline \multirow{2}{*}{\multicolumn{2}{|c|}{ Treatment }} & \multicolumn{8}{|c|}{ Days after planting (mean \pm S.E.) } \\
\hline & & 59 days & 66 days & 73 days & 80 days & 87 days & 94 days & 101 days & 108 days \\
\hline \multirow{4}{*}{$\begin{array}{l}\text { The length of } \\
\text { largest leaf } \\
(\mathrm{cm})\end{array}$} & $80 \mathrm{~g}$ & $78.0 \pm 2.0 \mathrm{a}$ & $78.0 \pm 2.0 \mathrm{a}$ & $78.0 \pm 2.0 \mathrm{a}$ & $78.0 \pm 2.0 \mathrm{a}$ & $78.0 \pm 2.0 \mathrm{a}$ & $78.0 \pm 2.0 \mathrm{a}$ & $78.0 \pm 2.0 \mathrm{a}$ & $78.0 \pm 2.0 \mathrm{a}$ \\
\hline & $120 \mathrm{~g}$ & $83.1 \pm 2.2 \mathrm{a}$ & $83.1 \pm 2.2 \mathrm{a}$ & $83.1 \pm 2.2 \mathrm{a}$ & $83.1 \pm 2.2 \mathrm{a}$ & $83.1 \pm 2.2 \mathrm{a}$ & $83.1 \pm 2.2 \mathrm{a}$ & $83.1 \pm 2.2 \mathrm{a}$ & $83.1 \pm 2.2 \mathrm{a}$ \\
\hline & $160 \mathrm{~g}$ & $84.6 \pm 1.6 \mathrm{ab}$ & $84.6 \pm 1.6 \mathrm{ab}$ & $84.6 \pm 1.6 \mathrm{ab}$ & $84.6 \pm 1.6 a b$ & $84.6 \pm 1.6 \mathrm{ab}$ & $84.6 \pm 1.6 a b$ & $84.6 \pm 1.6 \mathrm{ab}$ & $84.6 \pm 1.6 \mathrm{ab}$ \\
\hline & $200 \mathrm{~g}$ & $85.3 \pm 3.2 b$ & $5.3 \pm 3.2 b$ & $85.3 \pm 3.2 b$ & & & $85.3 \pm 3.2 b$ & & \\
\hline \multirow{4}{*}{$\begin{array}{l}\text { A number of } \\
\text { female flowers } \\
\text { (flower) }\end{array}$} & $80 \mathrm{~g}$ & $42.8 \pm 0.7 \mathrm{a}$ & $46.5 \pm 0.8 \mathrm{a}$ & $50.2 \pm 0.8 \mathrm{a}$ & $52.5 \pm 0.9 \mathrm{a}$ & $54.7 \pm 1.0 \mathrm{a}$ & $56.2 \pm 1.1 \mathrm{a}$ & $57.5 \pm 1.4 \mathrm{a}$ & $59.0 \pm 1.5 \mathrm{a}$ \\
\hline & $120 \mathrm{~g}$ & $43.6 \pm 0.4 a$ & $46.7 \pm 0.6 \mathrm{a}$ & $50.4 \pm 0.7 \mathrm{a}$ & $52.9 \pm 0.9 \mathrm{a}$ & $54.9 \pm 1.0 \mathrm{a}$ & $56.3 \pm 1.2 \mathrm{a}$ & $58.8 \pm 1.4 \mathrm{a}$ & $59.9 \pm 1.6 \mathrm{a}$ \\
\hline & $160 \mathrm{~g}$ & $45.4 \pm 0.4 \mathrm{ab}$ & $49.6 \pm 0.6 \mathrm{ab}$ & $53.2 \pm 0.7 \mathrm{ab}$ & $55.6 \pm 0.8 \mathrm{ab}$ & $58.0 \pm 1.0 \mathrm{ab}$ & $59.7 \pm 1.1 \mathrm{ab}$ & $61.3 \pm 1.3 \mathrm{ab}$ & $63.1 \pm 1.6 \mathrm{ab}$ \\
\hline & $200 \mathrm{~g}$ & $45.8 \pm 0.7 \mathrm{~b}$ & $50.1 \pm 0.7 \mathrm{~b}$ & $54.5 \pm 0.8 b$ & $57.2 \pm 0.7 \mathrm{~b}$ & $59.5 \pm 0.8 b$ & $61.8 \pm 0.9 b$ & $63.7 \pm 0.9 b$ & $66.1 \pm 1.3 \mathrm{~b}$ \\
\hline \multirow{4}{*}{$\begin{array}{l}\text { Total number } \\
\text { of flowers } \\
\text { (flower) }\end{array}$} & $80 \mathrm{~g}$ & $14.0 \pm 1.2 \mathrm{a}$ & $16.2 \pm 1.3 \mathrm{a}$ & $18.9 \pm 1.1 \mathrm{a}$ & $20.9 \pm 1.0 \mathrm{a}$ & $2.5 \pm 1.1 \mathrm{a}$ & $23.8 \pm 1.1 \mathrm{a}$ & $25.3 \pm 1.1 \mathrm{a}$ & $26.4 \pm 1.2 \mathrm{a}$ \\
\hline & $120 \mathrm{~g}$ & $14.8 \pm 0.7 \mathrm{ab}$ & $16.7 \pm 1.0 \mathrm{ab}$ & $19.3 \pm 1.0 \mathrm{ab}$ & $21.9 \pm 0.9 \mathrm{ab}$ & $23.9 \pm 0.7 \mathrm{ab}$ & $25.3 \pm 0.6 \mathrm{ab}$ & $26.6 \pm 0.4 \mathrm{ab}$ & $27.9 \pm 0.5 \mathrm{ab}$ \\
\hline & $160 \mathrm{~g}$ & $15.8 \pm 0.8 b$ & $18.0 \pm 1.2 \mathrm{~b}$ & $21.0 \pm 1.0 \mathrm{~b}$ & $23.3 \pm 0.9 b$ & $25.2 \pm 0.9 \mathrm{~b}$ & $26.8 \pm 0.8 \mathrm{ab}$ & $28.4 \pm 0.7 \mathrm{ab}$ & $29.9 \pm 0.7 \mathrm{ab}$ \\
\hline & $200 \mathrm{~g}$ & $14.8 \pm 1.0 \mathrm{ab}$ & $16.6 \pm 1.2 \mathrm{ab}$ & $20.2 \pm 1.2 \mathrm{ab}$ & $22.9 \pm 1.3 \mathrm{ab}$ & $24.7 \pm 1.3 \mathrm{ab}$ & $26.9 \pm 1.1 \mathrm{~b}$ & $28.6 \pm 1.1 \mathrm{~b}$ & $30.8 \pm 1.1 \mathrm{~b}$ \\
\hline \multirow{4}{*}{$\begin{array}{l}\text { The ratio of } \\
\text { female flowers } \\
\text { to the total } \\
\text { number of } \\
\text { flowers }(\%)\end{array}$} & $80 \mathrm{~g}$ & $32.5 \pm 2.3 \mathrm{a}$ & $34.6 \pm 2.3 \mathrm{a}$ & $37.6 \pm 2.0 \mathrm{a}$ & $39.8 \pm 1.7 \mathrm{a}$ & $41.1 \pm 1.7 \mathrm{a}$ & $42.3 \pm 1.5 \mathrm{a}$ & $44.0 \pm 1.5 \mathrm{a}$ & $44.7 \pm 1.5 \mathrm{a}$ \\
\hline & $120 \mathrm{~g}$ & $34.0 \pm 1.9 \mathrm{a}$ & $35.9 \pm 2.4 \mathrm{a}$ & $38.6 \pm 2.5 \mathrm{a}$ & $41.7 \pm 2.2 \mathrm{a}$ & $43.8 \pm 2.0 \mathrm{a}$ & $45.3 \pm 1.8 \mathrm{a}$ & $45.6 \pm 1.7 \mathrm{a}$ & $46.9 \pm 1.6 \mathrm{a}$ \\
\hline & $160 \mathrm{~g}$ & $34.9 \pm 2.0 \mathrm{a}$ & $36.3 \pm 2.4 \mathrm{a}$ & $39.6 \pm 2.1 \mathrm{a}$ & $42.0 \pm 2.0 \mathrm{a}$ & $43.6 \pm 2.0 \mathrm{a}$ & $45.1 \pm 1.8 \mathrm{a}$ & $46.5 \pm 1.6 \mathrm{a}$ & $47.6 \pm 1.4 \mathrm{a}$ \\
\hline & $200 \mathrm{~g}$ & $32.2 \pm 1.9 \mathrm{a}$ & $33.0 \pm 2.3 \mathrm{a}$ & $37.0 \pm 2.2 \mathrm{a}$ & $40.0 \pm 2.3 \mathrm{a}$ & $41.5 \pm 2.2 \mathrm{a}$ & $43.6 \pm 2.0 \mathrm{a}$ & $44.9 \pm 1.7 \mathrm{a}$ & $46.6 \pm 1.6 \mathrm{a}$ \\
\hline
\end{tabular}

The ratio of female flowers to the total number of flowers $=$ a number of female/total number of flowers $\times 100 ;{ }^{\mathrm{y}}$ Different letter indicate statistical differences between treatments were tested using the Turkey-Kramer test at $\mathrm{P}<0.05(\mathrm{n}=10)$. 
the volume of medium was $15 \mathrm{~L}$. Water retention and nutrient capacity of rice husk charcoal differ from those of peat, suggesting that the amount of applied fertilizer in the present study is slightly higher than that of Takemyou et al. (2002) [24].

Taken together, the optimum blend ratio of rice husk charcoal to peat for potted culture of zucchini is $80: 20$, and the optimum amount of fertilizer is $160-200 \mathrm{~g}$. In the future, we intend to examine the effect of timing of side dressing application on zucchini yield.

\section{REFERENCES}

[1] Powell, C.A., Stoffella, P.J. and Paris, H.S. (1993) Plant population influence on squash yield, sweetpotato whitefly, squash silverleaf, and zucchini yellow mosaic, HortScience, 28, 796-798.

[2] Ikeura, H., Tokuda, T. and Hayata, Y. (2012) Efficacy in zucchini potted culture. Journal of the Japanese Society of Agricultural Technology, 19, 7-11.

[3] Beardsell, D.V., Nichols, D.G. and Jones, D.L. (1979) Physical properties of nursery potting-mixtures. Scientia Horticulturae, 11, 1-8. doi:10.1016/0304-4238(79)90048-7

[4] Oshio, H., Nii, F. and Namioka, H. (1981) Characteristics of kuntan (rice husk charcoal) asoa mediumof soilless culture. Journal of the Japanese Society for Horticultural Science, 50, 231-238. doi:10.2503/jijshs.50.231

[5] Yoshida, Y., Hanaoka, T. and Hidaka, K. (2002) Effect of Composition of Nutrient Solution on Nutrient and Water Uotake, Growth and Yield of "Nyoho" Strawberry Growth with Peat Based Substrate. Horticultural Research, 1, 199-204. doi:10.2503/hrj.1.199

[6] Kato, H., Komori, A. and Miyake, H. (1996) Studies on nutrient solution culture of roses by drainage bed using rice husk charcoal as medium. Bulletin of Yamanashi Prefectural Agriculture Research Center, 7, 15-23.

[7] Sudhagar, R. and Sekar, K. (2009) Effect of coco peat medium on growth and quality of poinsettia (Euphorbia pulcherrima. Willd.). Asian Journal of Horticulture, 4, 52-56.

[8] Nazari, F., Farahmand, H., Khosh-Khui, M. and Salehi, H. (2011) Effects of coir as a component of potting media on growth, flowering and physiological characteristics of hyacinth (Hyacinthus orientalis L. cv. Sonbole-Irani). International Journal of Agricultural and Food Science, 1, 34-38.

[9] Vaughn, S., Deppe, N.A., Palmquist, D.E. and Berhow, M.A. (2011) Extracted sweet corn tassels as a reneable alternative to peat in greenhouse substrates. Industrial Crops Production, 33, 514-517. doi:10.1016/j.indcrop.2010.10.034

[10] Manios, T. (2004) The composting potential of different organic solid wastes: Experience from the island of Crete. Environment International, 29, 1079-1089. doi:10.1016/S0160-4120(03)00119-3

[11] Bari, Q.H. and Koenig, A. (2001) Effect of air recircula- tion and reuse on composting of organic solid waste. Resources Conservation and Recycling, 33, 93-111. doi:10.1016/S0921-3449(01)00076-3

[12] Promchot, S. and Boonprakob, U. (2007) Replacing agar with vermiculite, coconut fiber and charcoal rice husk in culture media for embryo rescue of immature nectarines seeds. Thai Journal of Agricultural Science, 40, 167-173.

[13] Miao, Y., Horibe, K., Hoki, M. and Iwai, S. (1999) Uses of rice husk charcoal for container culture. Bulliten Graduate School of Bioresorce Mie University, 23, 31-36.

[14] Inden, H. and Torres, A. (2004) Comparison of four substrates on the growth and quality of tomatoes. Acta Horoticultureae, 644, 205-215.

[15] Islam, S. (2008) Evaluating performance of ecologically sound organic substrates under different temperature regimes. International Journal of Agriculture and Biology, 10, 297-300.

[16] Endo, M., Kiriiwa, Y. and Nukaya, A. (2006) Effects of coir and peat ratios on growth, yield and water relations of strawberries "akihime" grown in soilless culture, Journal of the Japanese Society for Horticultural Science, 75, 344-349. doi:10.2503/jjshs.75.344

[17] Zaller, J.G. (2007) Vermicompost as a substitute for peat in potting media: Effects on germination, biomass allocation, yields and fruits quality of three tomato varieties Scientia Horticulturae, 112, 191-199. doi:10.1016/j.scienta.2006.12.023

[18] Wien, H.C., Stapleton, S.C., Maynard, D.N., Mc.Clurg, C. and Riggs, D.I.M. (2004) Flowering, sex expression, and fruiting of pumpkin (Cucurbita sp.) cultivars under various temperatures in greenhouse and distant field trials. HortScience, 39, 239-242.

[19] Zuraiqi, S. and Battikhi, A.M. (1992) The effect of Frequency of Nitrogen Application on growth yield and quality of tomato grown under plastic-house. Emir Journal of Agricultural Science, 4, 1-13.

[20] Diez, J.A., Roman, R., Caballero, R. and Caballero, A. (1997) Nitrate leaching from soils under a maize-wheatmaize sequence, two irrigation schedules and three types of fertilizers, Agriculture, Ecosystems \& Environment, 65, 189-199. doi:10.1016/S0167-8809(97)00045-5

[21] Eghball, B. and Power, J.F. (1999) Composted and noncomposted mature application to conventional and no-tillage systems: Corn yield and nitrogen uptake, Agronomy Journal, 91, 819-825. doi:10.2134/agronj1999.915819x

[22] Evanylo, G., Sherony, C., Spargo, J., Starner, D., Brosius, M. and Haering, K. (2008) Soil and water environmental effects of fertilizer-, manure-, and compost-based fertility practices in an organic vegetable cropping system. Agriculture, Ecosystems \& Environment, 127, 50-58. doi:10.1016/j.agee.2008.02.014

[23] Reiners, S. and Riggs, D.I.M. (1997) Plant spacing and variety affect pumpkn yield and fruit size, but supplemental nitrogen does not. HortScience, 32, 1037-1039.

[24] Takemyou, T., Araki, T., Nagatani, H., Tamaki, M. and Hayata, Y. (2002) Study on the potted culture of zucchini (Cucurbita pepo L.). Bulletin of Hiroshima Prefectural University, 13, 95-100. 